InnOvaciOnes de NegOciOs 18(35):

(C) 2021 UANL, Impreso en México (ISSN: 2007-1191)

Recepción: 13 de diciembre 2020 Aceptación: 7 de enero 2021

\title{
Actitudes emprendedoras que inciden en el desarrollo empresarial de los estudiantes universitarios de Instituciones de Educación Superior (IES), en México (Entrepreneurial Attitudes that Influence the Business Development of University Students of Higher Education Institutions (IES), in Mexico)
}

\author{
María Margarita Carrera Sánchez ${ }^{1}$, Abel Partida Puente ${ }^{2}$, Luis Alberto \\ Villarreal Villarreal ${ }^{3}$ \& Antonio Carlos Cantú Villarreal 4 \\ Universidad Autónoma de Nuevo León \\ maria.carrerasn@uanl.edu.mx 1, abel.partidapnt@uanl.edu.mx 2 , \\ luis.villarrealvl@uanl.edu.mx ${ }^{3}$, antonio.cantuvl@uanl.edu.mx ${ }^{4}$
}

\begin{abstract}
Entrepreneurship is a topic of interest and current, as well as entrepreneurial attitudes, which are a fundamental part of business development in students in Higher Education Institutions (IES), being a topic debated for some years by the majority of countries, this thanks to the great contributions in science and technology that they have achieved to date in the economic development of each country. The present research work aims to describe and analyze the entrepreneurial attitudes that affect the business development of university students in HEls. Implementing a statistical analysis through a multiple linear regression through the Statical Package for the Social Sciences (SPSS v18) software, by collecting a survey of 457 students from a Mexican university, distributed in the career of public accountant, graduated in administration, graduate in information technology and graduate in international business. The hypothesis raised in relation to entrepreneurial attitudes that positively affect business development is contrasted with the study population. The contributions of the activities carried out by the business development of the entrepreneurs favor the environment and contribute to the generation of jobs and economic development. In such a way that the entrepreneurial attitudes that were significant in the business development of university students are Achievement Motivation (ML), Entrepreneurship Motivation (MPE)
\end{abstract}

\section{Actitudes emprendedoras que inciden en el desarrollo empresarial}


and University Entrepreneurial Attitude (AEU), with a significance value below $5 \%$.

Keywords: attitudes, entrepreneurs and business management

JEL: J24, L26 y M13.

Resumen: El emprendimiento es un tema de interés y de actualidad, así como las actitudes emprendedoras, que son parte fundamental en el desarrollo empresarial en los estudiantes en las Instituciones de Educación Superior (IES), siendo un tema debatido desde hace algunos años por la mayoría de los países, esto gracias a los grandes aportes en la ciencia y tecnología que han alcanzado a la fecha en el desarrollo económico de cada país. El presente trabajo de investigación tiene como objetivo describir y analizar las actitudes emprendedoras que inciden en el desarrollo empresarial de los estudiantes universitarios en las IES. Implementando un análisis estadístico a través de una regresión lineal múltiple a través del software Statical Package for the Social Sciences (SPSS v18), mediante la recolección de una encuesta a 457 estudiantes de una universidad mexicana, distribuidos en la carrera de contador público, licenciado en administración, licenciado en tecnologías de información y licenciado en negocios internacionales. La hipótesis planteada en relación con las actitudes emprendedoras que inciden positivamente en el desarrollo empresarial es contrastada con la población de estudio. Las aportaciones de las actividades realizadas por el desarrollo empresarial de los emprendedores favorecen el entorno y contribuyen en la generación de empleos y desarrollo económico. De tal forma que las actitudes emprendedoras que fueron significativas en el desarrollo empresarial de los estudiantes universitarios son: la Motivación de Logro (ML), la Motivación por Emprender (MPE) y la Actitud Emprendedora Universitaria (AEU), con valor de significancia por debajo del $5 \%$.

Palabras clave: actitudes, emprendedores y gestión empresarial 


\section{Introducción}

En la actualidad las Instituciones de Educación Superior, están cada vez más comprometidas con el tema de emprendimiento por lo que constantemente se encuentran diseñando estrategias que permitan fortalecer a los jóvenes emprendedores que tienen intenciones de realizarse y alcanzar logros a través del emprendimiento empresarial. Para Leite, (2015) es relevante considerar los pilares básicos del desarrollo del espíritu emprendedor como es la educación, fuente innovadora inagotable de mejora e innovación. En tal sentido el informe del Monitor Global de Emprendimiento describe la referencia con la promoción del emprendimiento, para el 2017 México ocupaba el lugar 23 de entre 54 países. Aspectos como el financiamiento a emprendedores México ocupaba el lugar 33, así como los programas específicos de apoyo lugar 18, políticas de gobierno efectivas lugar 21 , e impuestos y burocracia lugar 26 , son datos considerados dentro del índice. Por lo que es interesante señalar que la actividad emprendedora en el país es ligeramente mayor que el promedio global de los países analizados. Por lo que preocupa que, para 2015, en la proporción de nuevos negocios que sobreviven más de 42 meses, México alcanzaba 6.86\% mientras que para el 2017 , este porcentaje cayó hasta $1.40 \%$. En cuanto a la tasa de innovación de los emprendedores, el país subió de $18.31 \%$ hasta un notable $31.71 \%$ con relación a los nuevos productos que son lanzados al mercado. El promedio global es de $26.50 \%$. El reporte de Global Entrepreneurship Monitor (GEM) llama la atención en cuanto a la baja educación en temas de emprendimiento en universidades, en financiamiento a nuevos negocios y la facilidad de hacer negocios como barreras al emprendimiento, aunque también reconoce que la

infraestructura física y el mercado interno han facilitado la actividad emprendedora.

En tal sentido la educación es una gran plataforma transformadora de la sociedad y que tiene alcance significativo a nivel social, nacional, regional o individual y será fruto de la educación (Leite, 2012).

El desarrollo de la cultura de emprendimiento ha resultado de vital importancia para impulsar el crecimiento económico de Latinoamérica, como lo muestra la fundación del instituto Global Entrepreneurship Monitor (GEM) con relación al emprendimiento en México que se ubicó en el lugar 56 de 126

\section{Actitudes emprendedoras que inciden en el desarrollo empresarial}


países analizados por el índice Global de Innovación en su reporte de 2018. Para describir el emprendimiento, el GEM, lo hace a partir de dos variables básicas, la actitud hacia el emprendimiento y las aspiraciones de los emprendedores. Este trabajo de investigación tiene como objetivo describir y analizar las actitudes emprendedoras que inciden en el desarrollo empresarial de los estudiantes universitarios en las IES.

Se realizó un estudio empírico entre los estudiantes de la Universidad Autónoma de Nuevo León, a través de la Facultad de Contaduría Pública y Administración. De acuerdo a la revisión de la literatura surge la siguiente pregunta de investigación ¿Cuáles son las actitudes emprendedoras que inciden en el desarrollo empresarial de los estudiantes universitarios en las Instituciones de Educación Superior?

La investigación se divide en seis secciones, iniciando con la sección I con el marco teórico considerando las principales conceptos y definiciones correspondientes a las variables y sus indicadores para lograr el sustento teórico de esta investigación, dentro de la sección II se analiza la parte metodológica en el cual se describe la estructuración del instrumento de investigación, ítem, variables, así como la muestra seleccionada para la aplicación del cuestionarían y el modelo de la ecuación diseñada, en la sección III se describen los resultados obtenidos con respecto a las significancias de las variables analizadas, el modelo, las correlaciones de cada una de las variables, en la sección IV se desarrolló la conclusión de la investigación con respecto a los resultados obtenidos y las variables analizadas, dentro de la sección $V$ se contempló la discusión de resultados haciendo un contrate entre los resultados obtenidos, investigaciones realizadas con estas variables y los principales aportaciones que tienen los autores con respecto a los resultados obtenidos, finalmente dentro de la sección VI se tienen las referencias bibliográficas en el cual se sustenta la información de los autores utilizados para el sustento teórico de esta investigación y de los resultados obtenidos. 


\section{Marco teórico}

\section{Actitudes emprendedoras}

Uno de los temas de gran interés en los últimos años es el emprendimiento, debido a la importancia que tiene en los procesos del desarrollo económico de los países, por tal motivo es trascendental los aspectos que contribuyen a que las ideas de emprendimiento puedan convertirse en realidades exitosas (Marulanda, Correa y Mejía, 2009). Por su parte Kirzner (1982), refiere el proceso emprendedor como un estado de alerta hacia las oportunidades basado en las diferencias en las percepciones individuales, y explica que el valor del emprendedor está en el aprovechamiento de oportunidades de beneficio sin explotar procedentes de una mala asignación de recursos. La Comisión Europea, en el referente Libro Verde (2003), define el espíritu empresarial como "la actitud y el proceso de crear una actividad económica combinando la asunción de riesgos, la creatividad y la innovación con una gestión sólida, en una organización nueva o en una ya existente". Para Leite. (2012) considera la actitud emprendedora como la principal entrada para la creación de empresas.

En tal sentido la motivación empresarial juego un papel muy importante ya que en la actualidad la motivación ya no se concibe como la satisfacción de necesidades, sino como la activación de conductas orientadas a fines y metas definidas por las características propias del individuo y de la coordinación social (Páez, Fernández, Basabe y Grad, 2002).

Desarrollo de Habilidades Empresariales

En cuanto a la relación de las actitudes emprendedoras en el desarrollo empresarial para Martínez y Campos (2014) describen que, aunque el desarrollo de una actitud favorable al emprendimiento no depende solo del sistema educativo, la parte universitaria de dicho sistema sí puede trabajar para intentar conformar una actitud como la buscada. Su objetivo sería aumentar el número de personas con suficiente conocimiento sobre el emprendimiento, la pequeña empresa y el autoempleo, de modo que puedan

\section{Actitudes emprendedoras que inciden en el desarrollo empresarial}


considerarlo una alternativa viable y racional para desarrollar su carrera profesional.

La Universidad, tiene la responsabilidad de desarrollar e incrementar la intención hacia el emprendimiento en sus estudiantes, ayudándolos a desarrollar habilidades, actitudes y comportamientos empresariales a través de diversas estrategias en el aula en donde se adquieran nuevos modelos de rol (Bertones y Radrigán, 2018).

En tal sentido, la educación emprendedora es una opción, ya que ésta se encamina a ofrecer a los estudiantes de cualquier nivel, el desarrollo de competencias, capacidades e instrumentos para que se forme como un actor de desarrollo económico y social, genera jóvenes independientes, innovadores, creativos, emprendedores auto motivados que cuestionen las normas establecidas y satisfagan sus metas personales a través de su propia acción (Espíritu, et. al., 2012).

La universidad fortalece el capital humano de sus estudiantes en dos niveles: la difusión de la cultura emprendedora y el desarrollo de competencias específicas para emprender (Caicedo, et al. 2015), sin duda, el fomento del emprendimiento dependerá de un sin número de factores en una nación, en este contexto, el clima o ecosistema emprendedor podría ser decisor al momento de construir un tejido emprendedor sostenible.

De tal forma que en un mundo en constante evolución y de incertidumbre económica, es necesario desarrollar una serie de capacidades, herramientas y actitudes para enfrentarse a las exigencias que demanda la nueva sociedad. Para ello, se debe poner el foco en el aprendizaje a lo largo de la vida y la adquisición de competencias emprendedoras, especialmente en el desarrollo de los programas de educación universitaria (European Higher Education Area, EHEA, 2012).

\section{Actitudes emprendedoras}

Las actitudes son los enunciados o juicios evaluadores de personas, objetos o eventos. Posee tres componentes: cognitivo -segmento de opinión o creencia-, afectivo -segmento emocional o sentimental- y comportamental intención de conducta de cierto modo hacia alguien o algo (Robbins \& Judge, 2011).

\section{Carrera, M.; Partida, A.; Villarreal, L. \& Cantú, A.}


La actitud emprendedora puede definirse como una conducta permanente de administrar los recursos para generar resultados según la actividad en que se desarrolla (Quintero, 2007).

Las principales actitudes emprendedoras objeto de nuestro estudio y que definiremos a continuación son: El Control Percibido Interno; La innovación; La Planeación y Visión del Futuro y La Motivación de Logros.

\section{Control Percibido Interno}

Por otra parte, para Martínez de Luco y Campos Granados (2014) señala el Control Percibido Interno, señala que lo importante es la percepción de la propia capacidad para llevar adelante la iniciativa emprendedora. Del mismo modo, se considera que la educación y la capacitación son algunos de los factores principales para fomentar el espiritu empresarial, ya que son un instrumento para crear iniciativas más desarrolladas y con mayores posibilidades de éxito (Coduras et al., 2008).

Así mismo, para que los emprendedores se sientan motivados por conseguir sus metas de negocio deben tener un control de las decisiones que toman para conseguir dichos objetivos (Espíritu y Sastre 2007).

Es importante enfatizar qué en el Control Percibido Interno, la persona debe creer que los resultados que obtiene en su vida son debidos a sí mismo (Santamaría, et al., 2017).

El control percibido interno se relaciona positivamente con: Esfuerzo en el trabajo, Nuevas ideas, con iniciativa, Negativamente con la creencia de la suerte, ideas viejas, decepción ante la no consecución de metas, obediencia, etc., Es más persuasiva porque busca más información y es más propensa a olvidar los recuerdos del fracaso. (Es muy importante que el emprendedor posea esta actitud, ya que a lo largo del emprendimiento tendrá fracasos que deberá afrontar.)

De forma más concreta, el emprendimiento puede favorecer el desarrollo de capacidades para crear un negocio social, apoyado en la creatividad, la autosuficiencia, el pensamiento racional, la empatía y las habilidades empresariales del alumnado, contribuyendo así, al desarrollo de una economía sostenible. Por ello, surge la necesidad de generar modelos

\section{Actitudes emprendedoras que inciden en el desarrollo empresarial}


educativos con una estructura de aprendizaje holístico que desarrolle aprendizajes cognitivos, conductuales y afectivos (Glunk y Van Gils, 2010).

En tal sentido, acaben o no estableciéndose por cuenta propia el aprendizaje del emprendimiento, esto beneficia a los jóvenes porque desarrollan conocimientos empresariales y aptitudes y actitudes esenciales (creatividad, imaginación, iniciativa, responsabilidad, trabajo en equipo, conocimiento del riesgo, entre otras) para el mercado laboral y para su empleabilidad (González, 2004).

\section{Innovación}

En cuanto a la innovación se refieren algunos aspectos que permiten caracterizar y distinguir los procesos emprendedores clave: Orientación a la innovación, Predisposición a asumir riesgos y Pro actividad.

Estos aspectos son los que definen la orientación emprendedora que sintetiza cómo se dirige el nuevo negocio. Se refiere a la puesta en marcha del espíritu emprendedor. El emprender explica en qué consiste este proceso. Es la creación de un nuevo negocio (Fernández, Alegre, y Chiva, 2012).

La autoeficacia emprendedora identifica la propia eficacia percibida (seguridad en uno mismo) para definir la idea de negocio y estrategia de la empresa, controlar el proceso de creación, negociar, reconocer oportunidades, y relacionarse con personas es clave para obtener capital.

Según los autores Martínez de Luco y Campos Granados (2014) el desarrollo de actitudes emprendedoras en el aula aporta nuevas capacidades en su desarrollo personal y profesional, alentándolos a descubrir, crear y explotar oportunidades.

Una persona emprendedora es aquella capaz de reconocer oportunidades con resolución y creatividad, siendo capaz de crear nuevas formas de hacer las mismas cosas, rompiendo con ello las ideas concebidas previamente. Son personas creativas, capaces de innovar y de enfrentar riesgos (Barrera, 2017).

Del mismo modo, el concepto de innovación también está muy relacionado con el emprendedurismo, porque son los emprendedores los que generan las iniciativas de innovación, utilizando sus capacidades y las aprendidas en redes de cooperación técnicas. Una de las principales

\section{Carrera, M.; Partida, A.; Villarreal, L. \& Cantú, A.}


limitaciones al emprendimiento es la falta de recursos económicos como capital semilla o de inversionistas interesados en la iniciativa, por lo que son sumamente relevantes las ayudas económicas públicas a la innovación.

Dentro del documento, El Libro Verde de la Comisión de las Comunidades Europeas (2003), se define el emprendimiento como un "modelo mental y el proceso de crear y desarrollar una actividad económica combinando con cierto talante gerencial, riesgo con creatividad y/o innovación, bien sea, en una organización nueva o en una existente".

La innovación, por su parte, se refiere a la "tendencia a comprometerse con y apoyar ideas nuevas, la novedad, la experimentación y los procesos creativos que pueden dar lugar a nuevos productos, servicios o procesos tecnológicos" (Bustos, 2015).

\section{La Planificación y Visión a Futuro}

Se postulan los siguientes argumentos:

Educación sobre emprendimiento: Enseñar a los estudiantes diferentes aspectos sobre la creación de empresas, sobre las actitudes y los valores propios de empezar una empresa, gerencial y hacerla crecer. La enseñanza es desde una perspectiva teórica.

Educación para emprender: Se trata de formar emprendedores que creen sus propias empresas. Se les enseña a preparar su propio plan de negocios.

Dado que la propensión de los estudiantes universitarios a crear una empresa como salida profesional no es alta, las universidades van incorporando, de forma creciente, en sus planes de estudios, materias específicas relacionadas con este tema. Para los autores Espíritu y Sastre (2007). Entre los nuevos objetivos de la educación superior se encuentran: Desarrollo de capacidades, adquiriendo competencias necesarias para promover a lo largo de toda la vida como: La creatividad, la flexibilidad, la capacidad de adaptación y la habilidad para aprender a emprender

En el contexto de la Teoría de la acción planificada, desarrollada por Ajzen en 1991. Se define, que la realización o no de una conducta está antecedida por nuestra intención para llevar a cabo dicha conducta. Esta intención se forma, entre otras cosas, por el aprendizaje e incorporación de

\section{Actitudes emprendedoras que inciden en el desarrollo empresarial}


experiencias propias, así como por las experiencias y actitudes de personas referentes para nosotros de nuestro entorno. Pero la decisión de crear una empresa no estaría solo influenciada por el proceso de socialización, sino también por otras variables del macro entorno que regularían, favoreciendo 0 disminuyendo la aparición de nuevos empresarios en sociedades o escenarios concretos.

En los últimos años, en Latinoamérica se ha dado mucha importancia a la formación en emprendimientos a través de cursos, centros de apoyo y asesoría, incubadoras, etc. (Espíritu, 2011). Llegando, la institucionalidad educativa, a formar un círculo virtuoso junto con el estado y demás instituciones relacionadas, quienes actúan en fomento y desarrollo de la actividad emprendedora, pudiendo afirmar que la intención y las aptitudes emprendedoras llegan a forjarse a través de la educación (Formichella, 2004).

A pesar de lo difícil que resulte ser innovador y lograr incursionar en el mercado, lo que verdaderamente importa es poseer una idea nueva de negocio, trascender de la idea a concepto de negocio, y arriesgarse, cambiando la rutina en el mercado (Hernández Morales, 2020).

En la actualidad, la formación universitaria implica generar propuestas orientadas al fomento del desarrollo económico local, por lo que "la utilización de las ideas apropiadas se convierte en la principal fuente de ventaja competitiva para los individuos y la organización en la época actual" (Cabrera, 2005). Por lo anterior, la creación de una empresa, es el resultado de la transformación de las ideas, considerando las condiciones del entorno y combinando la actitud emprendedora en la formación del estudiante Universitario.

\section{Motivación de Logro}

Para Espíritu y Sastre (2007) la motivación de logro se constituye como el deseo que tienen ciertas personas para mejorar el resultado de sus acciones y sentirse responsables de las mismas. Se ha relacionado con la probabilidad de convertirse en fundadores y de estar motivados para alcanzar el éxito. Según los autores:

La educación una mayor motivación hacia la consecución y logro de metas.

\section{Carrera, M.; Partida, A.; Villarreal, L. \& Cantú, A.}


Los hijos de padres empresarios tienen más probabilidades de convertirse en trabajadores por cuenta propia.

Los emprendedores requieren de información, asesoramiento y habilidades para emprender su actividad.

La percepción de un mayor apoyo social incide positivamente en una mayor intención de crear una empresa en universitarios.

En las familias emprendedoras todos los miembros deben ser formados de manera que sean capaces de generar sus propios negocios y no dependan exclusivamente del negocio fundado por sus padres (Ibarreche, 2019).

También es importante destacar la influencia que la Universidad puede tener en la motivación de los estudiantes, a través del impulso de los rasgos característicos de la actividad empresarial (motivación de logro, independencia, etc.), así como el apoyo institucional que puede proporcionar a los universitarios con inquietudes emprendedoras (orientación, asesoramiento, infraestructura, etc.) (Toledano, 2006).

Las intenciones emprendedoras han sido definidas en alusión a los estados mentales que se dirigen hacia: La atención, experiencia y acción hacia un concepto de negocio (Bird, 1988).

Se puede mencionar que la autoeficacia emprendedora, es definida como el conjunto de habilidades y destrezas que posee un sujeto, tanto cognitivas como conductuales, al igual que sus creencias, las cuales están influenciadas por diversos factores como la experiencia y las emociones, que determinarían el éxito o no ante la creación de una nueva empresa (Morales, et al., 2018).

El emprendimiento es una de las opciones para mejorar el nivel económico de la sociedad y es una de las opciones de futuro, por ello se hace necesario el trabajo de este desde todos los niveles de educación empezando por los más bajos y continuando hasta niveles superiores. Es, por tanto, una responsabilidad de los docentes el saber transmitir a su alumnado la importancia que tiene poseer una actitud emprendedora, trabajando el liderazgo, la creatividad y la autonomía (Barrera, 2017).

\section{Actitudes emprendedoras que inciden en el desarrollo empresarial}




\section{La Motivación para emprender}

En cuanto a la motivación para emprender, Toca (2010) señala como aquel ser humano que "asume riesgos, persigue algún beneficio; innova, identifica y crea oportunidades; establece y coordina nuevas combinaciones de recursos; y concibe nuevas formas de hacer las cosas".

Antes de crear una empresa se considera que debe existir en el individuo la intención de hacerlo Ajzen (1991); puntualiza acerca de la intención de una persona es el antecedente inmediato a su comportamiento. A partir de allí que pueda definirse para efectos de esta investigación a la intención emprendedora como una conducta planificada, orientada a la creación de una nueva empresa.

Por otra parte, las personas deben ser capaces de llevar a cabo diferentes aspectos que supone emprender: (según Martínez de Luco y Campos Granados, 2014), desde el reconocimiento de oportunidades, la definición de la idea de negocio, la puesta en marcha de la empresa, la negociación con inversores y entidades financieras.

El comportamiento planificado es intencional y, por lo tanto, se predice por la intención hacia ese comportamiento. En el modelo de Azjen (1991), la intención se predice por la actitud hacia el comportamiento o cuán atractivo es para el individuo ser un emprendedor; las normas subjetivas (NS) o la percepción de presión social para cumplir un comportamiento específico, y, el ACP o Auto - Capacidad Percibida.

Las principales características de los emprendedores son:

Están transformando el mundo (Timmons y Spinelli, 2007).

Ellos crean trabajo a partir de la innovación, reconocen oportunidades, aplican capital de riesgo y cambian la economía.

Utilizan el cambio como una oportunidad de mejora económica, no como un problema. Desde esta perspectiva, un emprendedor puede ser un gerente profesional o un colaborador cualquiera de la empresa.

Se necesita de la experimentación, de la imaginación, de la innovación para detectar oportunidades, haciendo que el emprendedor sea un factor clave en la empresa y en la sociedad, sobre la base del «saber y aprender a emprender» (Bueno, 2003).

\section{Carrera, M.; Partida, A.; Villarreal, L. \& Cantú, A.}




\section{Actitud Emprendedora Universitaria}

La importancia de la universidad en la formación de los estudiantes, para fortalecer y desarrollar el espíritu emprendedor, fomentar la cultura empresarial y contribuir con programas de estudio, así con docentes expertos en el ámbito empresarial.

Para Benavides et al., (2004); describe el papel de la Universidad en el proceso de generación de nuevos emprendedores no debería limitarse a la mera formación académica, sino que le correspondería ir más allá. Así, entre los nuevos objetivos de la educación superior debe encontrarse:

Desarrollo de la capacidad de empleo a través de la adquisición de competencias necesarias para promover, la creatividad, la flexibilidad, capacidad de adaptación, así como la habilidad para aprender a aprender y resolver problemas

En la actualidad, la formación universitaria implica generar propuestas orientadas al fomento del desarrollo económico local, por lo que "la utilización de las ideas apropiadas se convierte en la principal fuente de ventaja competitiva para los individuos y la organización en la época actual" (Cabrera, 2005). Por lo anterior, la creación de una MIPYME, es el resultado de la transformación de las ideas, considerando las condiciones del entorno y combinando la actitud emprendedora en la formación del estudiante Universitario.

La Comisión Europea (2013) reconoce la importancia y el impacto que tiene el espíritu empresarial en las instituciones de educación superior y desarrolla el Plan de Acción sobre Emprendimiento 2020. Su objetivo es facilitar la creación de empresas y crear un entorno mucho más favorable para que los emprendedores actuales puedan prosperar y crecer. El Plan propone tres áreas, entre las cuales destaca el importante papel de la educación y la formación, así como el de la propia universidad como generador de conocimiento innovador.

La educación universitaria debe incluir también la formación en las dimensiones más fundamentales asociadas al "saber ser", a la definición de la personalidad y al aprendizaje del liderazgo. En este sentido (Lazear, 2003; Pleitner, 2003) las universidades juegan un importante papel en el

\section{Actitudes emprendedoras que inciden en el desarrollo empresarial}


adiestramiento de emprendedores como individuos de características múltiples (citado en Martín, et al 2005).

El comportamiento emprendedor es algo que se está demandando de forma creciente en el contexto actual, y, por tanto, el desarrollo de competencias emprendedoras entre las graduadas y los graduados universitarios es una función para considerar por las instituciones de educación superior, así como por parte tanto del sector empresarial como de otro tipo de empleadores (Gibb, Haskins y Robertson, 2009).

En otro orden de ideas, "La Resiliencia como componente de la Actitud Emprendedora de los Jóvenes Universitarios" realizada por Fontaines, Palomo y Velásquez (2015), con 1,072 alumnos de la Universidad de Oriente Núcleo Monagas. Estos autores, observaron que la resiliencia representa un factor primordial para alcanzar las metas de emprendimiento, particularmente cuando está asociada con las variables: ser miembro de una familia con negocio propio, pertenecer a organizaciones comunitarias, tener empleo por cuenta propia y trabajar antes de culminar la carrera.

Otro factor importante, lo constituyen los planes de estudio de las distintas universidades y las características propias de cada institución educativa y de sus alumnos pueden incidir en las opciones de salida laboral evaluadas por sus graduados (Charney y Libecap, 2000). Por lo tanto, puede argumentarse que la naturaleza de la universidad y las características de la carrera dictada pueden influir sobre la vocación emprendedora del alumno.

En tal sentido Delicio (2007), señala que a la universidad compete diseñar planes de estudio que fomenten de manera integral la actitud emprendedora, donde se generalicen las prácticas de extensión universitaria, tanto para docentes como alumnos, en temas de apoyo a emprendedores".

\section{Actitud de Gestión Empresarial}

En los últimos años el tema de emprendimiento es determinante para el autoempleo y generación de este. Por lo que en la actualidad los gobiernos contemporáneos han comenzado a reivindicar el potencial de la iniciativa emprendedora como fuente de empleo, riqueza y bienestar social en las economías modernas. (Lanero, et al., 2015).

\section{Carrera, M.; Partida, A.; Villarreal, L. \& Cantú, A.}


Con la presente crisis global que ha sido reconocida por los diversos gobiernos del mundo y que se confirma en el informe de la Organización para la Cooperación y el Desarrollo Económico (OCDE), en donde se impone el reconocimiento del emprendimiento como una alternativa viable a las altas tasas de desempleo, pobreza y el desbalance entre el acceso a los recursos por parte de los diversos grupos sociales en el mundo (OCDE, 2013).

En tal sentido, Mol \& Yue (2015) consideran la promoción del espíritu empresarial y la innovación en la educación superior, que no solo se relaciona con la forma en cómo se encuentra estructurado el plan de estudios, se involucra también con la estructura del gobierno de la universidad y el liderazgo con el que se toman las decisiones (citado en Sánchez, 2016).

\section{Método}

El presente estudio es de tipo exploratorio, descriptivo, correlacional y explicativo con técnicas cuantitativas y cualitativas, de tipo correlacional puesto que se realiza un análisis de la relación entre variables donde se evalúa el grado de relación entre ellas midiendo en primer lugar cada una de estas variables, las cuantifica, analiza y establece vinculaciones, además estas correlaciones se sustentan en hipótesis que son sometidas a prueba (Hernández, et al., 2014). Sobre lo expuesto anteriormente, se estudian las actitudes emprendedoras que inciden en el desarrollo de habilidades emprendedoras en escuelas públicas de educación superior.

Para la recolección de datos se aplicó en junio-julio 2020 a través de una herramienta electrónica MS FORMS, el instrumento de medición estructurado en tres secciones: la primera con 14 datos que integran el perfil del estudiante en la segunda las actitudes con 41 y en la tercera desarrollo de habilidades empresariales con 7 datos en una escala tipo Likert asignado valores del 1 al 5 de la siguiente manera: (5) Totalmente de acuerdo, (4) De acuerdo, (3) Ni de acuerdo ni en desacuerdo, (2) En desacuerdo y (1) Totalmente en desacuerdo, donde se recolectan las percepciones de los estudiantes de estudiantes de séptimo a décimo semestre de las carreras de Contador Público, Licenciado en Administración, Licenciado en Tecnologías de Información y Licenciado en Negocios Internacionales, la Facultad de Contaduría Pública y Administración de la UANL. Determinándose una

\section{Actitudes emprendedoras que inciden en el desarrollo empresarial}


muestra representativa mediante el método de muestra probabilística estratificada. (Levine, et al., 2006).

El tamaño de la muestra fue estimada con la población finita de 20,500 estudiantes de la escuela pública de educación superior, con un error del $5 \%$ y un valor de p del $50 \%$, con un 0.95 del nivel de confianza; el resultado arrojó una tamaño óptimo de muestra de $n=370$, logrando aplicar un total de 457 encuestas.

Para ello se utilizó la fórmula siguiente:

$$
n=\frac{N z^{2} p(1-p)}{(N-1) e^{2}+z^{2} p(1-p)}
$$

$Z=$ Representa el nivel de confianza requerido.

$p=$ Proporción de personas cuya factibilidad de ser seleccionadas en la muestra es un éxito.

$\mathrm{N}=$ Población total sujeta de estudio.

$\mathrm{e}=$ Error de muestreo.

\section{Modelo}

El modelo del presente trabajo de investigación se estructuró con base a estudios empíricos, diseñado por Robinson en 1987, el "Entrepreneaurship Attitude Orientation Scale" (EAO) en los Estados Unidos y posteriormente traducido y modificado en España por lbañez en el 2001. (citado en Krauss, 2005) adaptando el instrumento a las necesidades del presente estudio la dimensión de las actitudes emprendedoras, para medir cómo las variables independientes de Control Percibido Interno (CPI), Innovación (I), Planificación y Visión del Futuro (PVF), Motivación de Logro (ML), integrando las variables de Motivación para Emprender (MPE), Actitud Emprendedora Universitaria (AEU), Actitud de Gestión Empresarial (AGE), inciden en la variable dependiente Desarrollo de Habilidades Empresariales (DHE) utilizadas por (Sánchez, et al, 2016). Estos ítems representan el análisis en la revisión de la literatura de acuerdo a trabajos de investigación previos, sobre las variables de estudio con escalas validadas en los artículos empíricos que le dan la validez y confiabilidad al instrumento aplicado

\section{Carrera, M.; Partida, A.; Villarreal, L. \& Cantú, A.}


El modelo que se estima para analizar las actitudes emprendedoras que inciden en el desarrollo empresarial de los estudiantes universitarios es el siguiente:

DHEi $=\beta 0+\beta 1 C P I$ Control Percibido Interno $+\beta 2$ I Innovación $+\beta 3$ PVF Planeación y Visión de Futuro + $\beta 4 \mathrm{ML}$ Motivación de Logro + $\beta 5$ MPE Motivos por Emprender + $\beta 6$ AEU Actitud Emprendedora Universitaria + $\beta 7$ AGE Actitud de Gestión Empresarial + $\beta 8$ AGE 1 Actitud de Gestión Empresarial +e

Donde:

Desarrollo de Habilidades Empresariales (DHE): Son las capacidades que integran los conocimientos, habilidades y actitudes que le permiten al ser humano desempeñarse de una manera autónoma.

Control Percibido Interno (CPI): La percepción de la propia capacidad interna para llevar adelante la iniciativa emprendedora. Martínez de Luco y Campos Granados (2014).

Innovación (I): se trata de una actitud imaginativa y creativa que facilita el funcionamiento de las cosas, la satisfacción de necesidades y la búsqueda de respuestas nuevas a problemáticas planteadas. Durán-Aponte y AriasGómez (2015).

Planeación y Visión de Futuro (PVF): Capacidad para diseñar y estructurar un proyecto de vida y/o empresarial.

Motivación de Logro (ML): la necesidad continua que experimenta la persona de alcanzar unas metas que la misma se ha fijado y de cuyos resultados se siente responsable (McClelland, 1968; Fernández y Junquera, 2001).

Motivos por Emprender (MPE): Factores que integran los motivos por emprender, la familia, los maestros, la escuela y tener la capacidad para identificar oportunidades.

Actitud Emprendedora Universitaria (AEU): Percepción en su formación, en la escuela en relación con las asignaturas, los maestros y el ambiente escolar fomenta la cultura emprendedora.

Actitud de Gestión Empresarial AGE): Conocimientos de los programas y apoyos económicos para emprender.

\section{Actitudes emprendedoras que inciden en el desarrollo empresarial}




\section{Resultados}

De la recolección de datos de los estudiantes participantes que respondieron el cuestionario, el $58 \%$ son mujeres y el $42 \%$ hombres; la edad promedio de los jóvenes fue de 21.72 años. El $82.3 \%$ de los estudiantes pertenecen al turno nocturno y el $8.4 \%$ al turno mixto, un $4.8 \%$ turno vespertino y el $4.5 \%$ al matutino. Recibiendo una mayor participación de encuestas de estudiantes de noveno semestre con el $51.1 \%$, el $24.3 \%$ de octavo semestre, el $16.3 \%$ de séptimo y el $8.3 \%$ de décimo semestre. En cuanto a la participación de envío de encuestas la carrera de Licenciado en Negocios Internacionales se recolectó el $26.25 \%$, Licenciado en Tecnologías de Información con el 26.06\%, Licenciado en Administración el $25.38 \%$ y de Contador Público un $22.31 \%$.

Respecto al ítem de cuál sería su trabajo ideal se encontró que el $54.71 \%$ desea tener su propia empresa, el $16.98 \%$ crear su propia empresa, el $13.20 \%$ eligió ser asalariado en una empresa multinacional, el $9.43 \%$ ser alto directivo, el $3.77 \%$ ser funcionario público y el $1.88 \% .9 \%$ asalariado en una pequeña empresa. Se realizó un análisis para identificar el nivel de escolaridad de los padres de familia que tienen negocio, con el ítem de cuál sería su trabajo ideal de los estudiantes y los hallazgos encontrados son que el $37 \%$ tiene estudios de licenciatura, el $23.2 \%$ tiene preparatoria, el $16.2 \%$ secundaria, el $14.4 \%$ tiene estudios de maestría, el $6.48 \%$ primaria y tan solo el $2.7 \%$ corresponde a otros. Respecto al ítem de cuál sería su trabajo ideal se encontró que el $58.5 \%$ desea tener su propia empresa, el $16.2 \%$ crear su propia empresa, con el mismo porcentaje $11.71 \%$ de representatividad, alto directivo y asalariado en una empresa multinacional, el . $9 \%$ desea ser funcionario público y el $.9 \%$ asalariado en una pequeña empresa.

A los estudiantes se les indago acerca de cuál es su trabajo ideal y evaluarse con relación a sus capacidades para ser empresario, y los hallazgos encontrados son que el $48.3 \%$ desea tener su propia empresa, el $18.1 \%$ crear su propia empresa, el $15.5 \%$ quiere ser un alto directivo, mientras que el $10.2 \%$ elige ser asalariado, el $3.06 \%$ desea ser funcionario público y el $3.06 \%$ asalariado en una pequeña empresa, y el .6\% se considera pesimista sobre sus opciones del futuro y el .87 corresponde a la opción de otros. En cuanto a su percepción de sus capacidades para ser empresario se encontró que el

Carrera, M.; Partida, A.; Villarreal, L. \& Cantú, A. 
$39.38 \%$ considera que tiene lo necesario para ser empresario, aunque debería de completar su formación práctica, el $19.7 \%$ considera que aunque no tenga todas las capacidades las puedo complementar con otras personas, el $17.94 \%$ considera que es completamente capaz, mientras que el $11 \%$ percibe que tiene lo necesario aunque debería de completar su formación teórica, el $4.6 \%$ desconoce cuáles son las capacidades para ser empresarios, el $4.59 \%$ percibe que sus capacidades para ser empresario son escasas, el $1.75 \%$ considera que ser empresario no necesita ninguna capacidad especial y el $.87 \%$ considera que no tiene ninguna capacidad para ser empresario.

Respecto a los familiares empresarios se encontró que el $63.9 \%$ corresponde a un tío, el $25.3 \%$ al padre, el $23.7 \%$ a un primo, el $10.8 \%$ a la madre, el $8.7 \%$ al abuelito y el $6.2 \%$ corresponde que al hermano.

Para efectos de validar el presente trabajo de investigación de los coeficientes alfas de Cronbach se utilizó el software SPSS, (véase tabla 1) de los constructos independientes de esta investigación: Control Percibido Interno (CPI), Innovación (I), Planificación y Visión del Futuro (PVF), Motivación de Logro (ML), Motivación por Emprender (MPE), Actitud Emprendedora Universitaria (AEU), Actitud de Gestión Empresarial (AGE)y de la variable dependiente Desarrollo de Habilidades Empresariales (DHE), las cuales se encuentran por arriba del mínimo aceptable de 0.650 .

Tabla 1. Coeficientes alfas de Cronbach

\begin{tabular}{lcc}
\hline CONSTRUCTOS & ALFA DE CRONBACH & NO. ITEMS \\
INDEPENDIENTES & & \\
\hline CPI & 0.770 & 6 \\
I & 0.775 & 6 \\
PVF & 0.664 & 5 \\
ML & 0.694 & 3 \\
MPE & 0.679 & 7 \\
AEU & 0.897 & 8 \\
AGE & 0.769 & 6 \\
CONSTRUCTO DEPENDIENTE & ALFA DE CRONBACH & NO.ITEMS \\
DHE & 0.794 & 7 \\
\hline
\end{tabular}

Fuente: Elaboración propia.

Estudio de la regresión lineal múltiple con la variable dependiente Desarrollo de Habilidades empresariales.

\section{Actitudes emprendedoras que inciden en el desarrollo empresarial}


Los constructos de estudio independientes se analizaron con una muestra de 457 encuestas de satisfacción con respecto al constructo dependiente para diseñar y analizar la regresión lineal múltiple. Los valores de la encuesta fueron introducidos al software estadístico SPSS-V18. Una vez introducidos y analizados en el software estadístico, se puede ver la linealidad (véase tabla 2) de acuerdo a Schwab (2007), el cual indica que correlaciones por arriba del 0.50 entre los constructos es muestra de linealidad, nos valida que la relación que existe entre el constructo dependiente: Desarrollo de Habilidades Empresariales (DHE) y los constructos independientes: Control Percibido Interno (CPI), Innovación (I), Planificación y Visión del Futuro (PVF), Motivación de Logro (ML), Motivación por Emprender (MPE), Actitud Emprendedora Universitaria (AEU), Actitud de Gestión Empresarial (AGE) es significativo en el rango de todos los valores.

Tabla 2. Correlaciones Pearson

\begin{tabular}{cccccccccc}
\hline & & \multicolumn{7}{c}{ Correlations } \\
\hline & & DHE & CPI & I & PVF & ML & MPE & AEU & AGE \\
& DHE & 1.000 & .323 & .355 & .320 & .119 & .673 & .803 & .477 \\
& CPI & .323 & 1.000 & .726 & .726 & .697 & .598 & .439 & .322 \\
Pearson & I & .355 & .726 & 1.000 & .634 & .528 & .648 & .419 & .439 \\
Correlation & ML & .320 & .726 & .634 & 1.000 & .646 & .543 & .424 & .295 \\
& MPE & .673 & .697 & .528 & .646 & 1.000 & .456 & .244 & .053 \\
& AEU & .803 & .439 & .648 & .543 & .456 & 1.000 & .794 & .516 \\
& AGE & .477 & .322 & .439 & .424 & .244 & .794 & 1.000 & .503 \\
& DHE &. & .000 & .000 & .000 & .005 & .516 & .503 & 1.000 \\
& CPI & .000 &. & .000 & .000 & .000 & .000 & .000 & .000 \\
Sig. (1- & I & .000 & .000 &. & .000 & .000 & .000 & .000 & .000 \\
tailed) & PVF & .000 & .000 & .000 &. & .000 & .000 & .000 & .000 \\
& ML & .005 & .000 & .000 & .000 &. & .000 & .000 & .129 \\
& MPE & .000 & .000 & .000 & .000 & .000 &. & .000 & .000 \\
& AEU & .000 & .000 & .000 & .000 & .000 & .000 &. & .000 \\
& AGE & .000 & .000 & .000 & .000 & .129 & .000 & .000 &. \\
& DHE & 457 & 457 & 457 & 457 & 457 & 457 & 457 & 457 \\
& CPI & 457 & 457 & 457 & 457 & 457 & 457 & 457 & 457 \\
N & I & 457 & 457 & 457 & 457 & 457 & 457 & 457 & 457 \\
& PVF & 457 & 457 & 457 & 457 & 457 & 457 & 457 & 457
\end{tabular}

Carrera, M.; Partida, A.; Villarreal, L. \& Cantú, A. 
Fuente: Elaboración propia.

\begin{tabular}{ccccccccc} 
ML & 457 & 457 & 457 & 457 & 457 & 457 & 457 & 457 \\
MPE & 457 & 457 & 457 & 457 & 457 & 457 & 457 & 457 \\
AEU & 457 & 457 & 457 & 457 & 457 & 457 & 457 & 457 \\
AGE & 457 & 457 & 457 & 457 & 457 & 457 & 457 & 457 \\
\hline
\end{tabular}

Una vez generadas las variables con promedios ponderados y ejecutada la regresión lineal de los constructos independientes, Control Percibido Interno (CPI), Innovación (I), Planificación y Visión del Futuro (PVF), Motivación de Logro (ML), Motivación por Emprender (MPE), Actitud Emprendedora Universitaria (AEU), Actitud de Gestión Empresarial (AGE) con respecto a al constructo dependiente Desarrollo de Habilidades Empresariales (DHE) se generaron los siguientes resultados.

La independencia de residuales a través de la Durbin-Watson, la cual muestra la presencia de auto correlación de primer orden en los residuales en una regresión, el valor Durbin-Watson generado es un valor de 2.015 asegurando que no existe auto correlación en el modelo mostrando una regresión lineal valida (rango permitido 1.5 a 2.5), con coeficiente de correlación aceptadas $(r)$ de 0.816 al igual que el coeficiente de determinación $\left(r^{2}\right)$ de 0.665 (véase tabla 3 ).

Tabla 3. Resultados de la regresión lineal

\begin{tabular}{|c|c|c|c|c|c|c|c|c|c|c|}
\hline \multicolumn{11}{|c|}{ Model Summaryb } \\
\hline \multirow[b]{2}{*}{$\begin{array}{c}\text { Mod } \\
\text { el }\end{array}$} & \multirow[b]{2}{*}{$R$} & \multirow[b]{2}{*}{$\begin{array}{c}\text { R } \\
\text { Squa } \\
\text { re }\end{array}$} & \multirow[b]{2}{*}{$\begin{array}{c}\text { Adjust } \\
\text { ed R } \\
\text { Squar } \\
\text { e }\end{array}$} & \multirow[b]{2}{*}{$\begin{array}{l}\text { Std. } \\
\text { Error } \\
\text { of the } \\
\text { Estima } \\
\text { te }\end{array}$} & \multicolumn{5}{|c|}{ Change Statistics } & \multirow[b]{2}{*}{$\begin{array}{c}\text { Durbi } \\
\text { n- } \\
\text { Wats } \\
\text { on }\end{array}$} \\
\hline & & & & & $\begin{array}{c}R \\
\text { Squar } \\
\text { e } \\
\text { Chan } \\
\text { ge }\end{array}$ & $\begin{array}{c}\text { F } \\
\text { Chan } \\
\text { ge }\end{array}$ & $\begin{array}{c}\mathrm{df} \\
1\end{array}$ & $\begin{array}{l}\text { df } \\
2\end{array}$ & $\begin{array}{c}\text { Sig. F } \\
\text { Chan } \\
\text { ge }\end{array}$ & \\
\hline 1 & $\begin{array}{l}.81 \\
66^{a}\end{array}$ & .665 & .660 & .48808 & .665 & $\begin{array}{c}127.4 \\
42\end{array}$ & 7 & $\begin{array}{c}44 \\
9\end{array}$ & .000 & 2.015 \\
\hline
\end{tabular}

a. Predictors: (Constant), AGE, ML, AEU, I, PVF, CPI, MPE

b. Dependent Variable: DHE

Fuente: Elaboración propia.

\section{Actitudes emprendedoras que inciden en el desarrollo empresarial}


Un modelo no debe reflejar multicolinealidad entre los constructos independientes por los cual podemos observar que no se muestra multicolinealidad entre los constructos independientes mostrando una colinealidad aceptable mediante el índice de factor de inflación de varianza (VIF) debido a que son menores a 10 (véase tabla 4).

Tabla 4. Índice de factor de inflación de varianza para ACTITUD

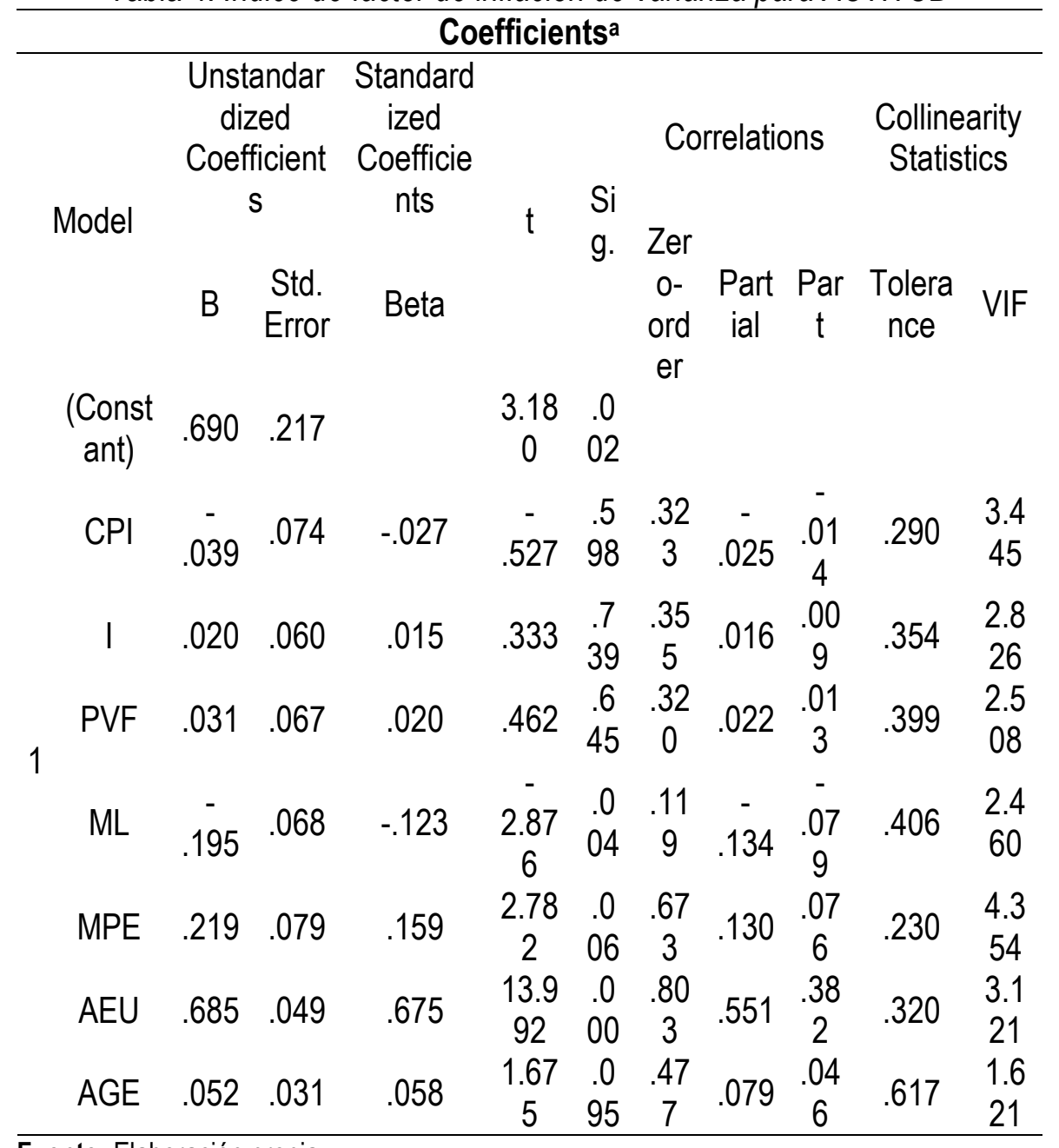

Fuente: Elaboración propia. 
Estudio de la T-student

Podemos concluir de acuerdo a las significancias de la T-student considerando un error estimado menor al $5 \%$ (ver tabla 4), que el modelo es aceptable, debido a que los constructos independientes que impacta significativamente al constructo dependiente Desarrollo de Habilidades Empresariales (DHE) son: Motivación de Logro (ML), Motivación por Emprender (MPE) y Actitud Emprendedora Universitaria (AEU), con valor de significancia por debajo del $5 \%$, excluyendo las variables independientes Control Percibido Interno (CPI), Innovación (I), Planificación y Visión del Futuro (PVF) y la variable independiente Actitud de Gestión Empresarial (AGE), por lo cual podemos diseñar la ecuación lineal que representa los resultados de esta investigación con la variable dependiente Desarrollo de Habilidades Empresariales (DHE).

$$
\hat{Y}=0.690-(0.195) \mathrm{ML}+(0.219) \mathrm{MPE}+(0.685) \mathrm{AEU}+\varepsilon
$$

\section{Discusión}

Una vez que se obtuvieron los resultados podemos concluir que, de los constructos independientes involucrados en la presente investigación, se encontró que en la dimensión de actitudes emprendedoras la variable de motivación de logro incide negativamente con un 19.5\% con relación en el desarrollo de habilidades empresariales, mientras que la motivación por emprender impacta significativamente con un $21.9 \%$ y la actitud emprendedora universitaria con un $68.5 \%$ en el desarrollo de habilidades empresariales en los estudiantes.

La aportación del trabajo de estudio describe la percepción de los estudiantes en función de las actitudes emprendedoras que fortalecen su formación académica para el desarrollo de habilidades empresariales, en el que se identifica que las actitudes no son significativas para emprender, por lo que es conveniente integrar en el modelo educativo asignaturas que fortalezcan las actitudes emprendedoras de los estudiantes con relación al Control Percibido Interno (CPI), Innovación (I), Planeación y Visión de Futuro (PVF) y Motivación de Logro (ML).

\section{Actitudes emprendedoras que inciden en el desarrollo empresarial}


Es importante destacar, en cuanto a la variable actitud de gestión empresarial que los estudiantes refieren desconocer los apoyos económicos, los programas, así como las publicaciones de las convocatorias para acceder a los apoyos que otorga el gobierno federal para los emprendedores. De tal forma que se perciben limitantes en la relación gobierno escuela para la divulgación de los programas de apoyo, así como nutrir, fortalecer y reforzar los programas académicos en las asignaturas vinculadas al desarrollo empresarial.

En cuanto a la variable de actitud emprendedora universitaria, los resultados son muy significativos ya que los estudiantes perciben que las asignaturas cursadas en la carrera a la fecha influyen en como emprender y desarrollan el espíritu emprendedor, de igual manera que sus maestros contribuyen al desarrollo de habilidades empresariales necesarias para generar productos y/o servicios nuevos. Así como también identifican que la universidad fomenta la cultura emprendedora a través de ferias, y concursos. Por lo que el trabajo de estudio difiere con Sánchez, et al, (2016) en relación con los estudiantes de la carrera de Ingeniería de Gestión Empresarial, que no perciben que la iniciativa empresarial y el espíritu emprendedor sean aprendidos o en su caso desarrollados.

Es importante seguir estudiando los constructos independientes de la dimensión de actitudes emprendedoras, Control Percibido Interno (CPI), Innovación (I), Planificación y Visión del Futuro (PVF) y la variable independiente Actitud de Gestión Empresarial (AGE) como futuras líneas de investigación para seguir incrementando aportaciones prácticas en los estudiantes.

Discusión de los resultados

En este trabajo se ha examinado las actitudes emprendedoras que inciden en el desarrollo empresarial de los estudiantes universitarios. Para lo anterior, se realizó una revisión de literatura y se utilizó el software SPSS para validar el modelo a través de las significancias de la T-student considerando un error estimado menor al 5\% (ver tabla 4) debido a que los constructos independientes que impacta significativamente al constructo dependiente Desarrollo de Habilidades Empresariales. El hallazgo más significativo es que,

\section{Carrera, M.; Partida, A.; Villarreal, L. \& Cantú, A.}


en la dimensión de las actitudes emprendedoras tuvo incidencia solo la variable motivación de logro, que resultó tener un signo negativo para la variable desarrollo de habilidades empresariales. Por lo que esto nos lleva a comprobar que los estudiantes que asumen mayor motivación de logro o que están más satisfechos con sus logros tienen una menor probabilidad de poseer una actitud para emprender. De igual forma Zapinco et. al (2008) plantea que las políticas de estímulo de la actividad emprendedora deberían tener en cuenta que no solamente las personas con actitudes negativas hacia el autoempleo rechazan crear empresas.

Un dato notable está relacionado con los padres de familia que tienen negocio propio, los estudiantes refieren el grado de escolaridad de sus padres es licenciatura y se encontró que los alumnos que sus padres tienen negocio propio aspiran a tener y crear su propia empresa. En esta perspectiva Garavan y O'Cinneide (1994), determinan que, para tener una mayor predisposición a ser emprendedor, es deseable descender de padres creadores de empresa con conocimientos y éxito, así como, tener experiencia laboral y una adecuada educación académica. Como puede inferirse, parece que el convertirse en emprendedor depende de varios factores

En tal sentido, Barba-Sánchez y Atienza-Sahuquillo (2012) definen que la decisión de creación y puesta en marcha de una empresa se ve como el producto de los motivos e intenciones que presenta la persona de acuerdo a sus particulares rasgos de personalidad y habilidades empresariales. En este sentido, Rubio (2012) señala que es necesario desarrollar actitudes y habilidades como la inteligencia de mercado, la valoración empresarial y el estímulo a instituciones educativas que fomenten la cultura en la opción del trabajo por cuenta propia. Por lo que es indispensable la colaboración de diversos agentes, incluida la administración pública y las organizaciones educativas.

Evidentemente, esto contribuye con lo expuesto por Postigo \& Tamborini (2005) que en los últimos años varios países han desarrollado sistemas educativos que no han favorecido el fomento del espiritu empresarial, por lo que es complicado hallar universidades que enseñen y forjen ese ánimo en sus estudiantes (en Sánchez, et al., 2016).

\section{Actitudes emprendedoras que inciden en el desarrollo empresarial}




\section{Referencias}

Ajzen, I. (1991). The theory of planned behavior. Organizational behavior and human decision processes, 50(2):179-211.

Barba-Sánchez, V. y Atienza-Sahuquillo, C. (2012). Entrepreneurial behavior: Impact of motivation factors on decision to create a new venture. Investigaciones Europeas de Dirección y Economía de la Empresa, 18. 132-138.

Barrera, E. (2017). Actitudes y orientación emprendedora de estudiantes universitarios: un estudio exploratorio en la Facultad de Ciencias de la Educación. Trabajo Fin de Máster Inédito Universidad de Sevilla.

Benavides, M., Sánchez, M., y Luna, R. (2004). El proceso de aprendizaje para los emprendedores en la situación actual: Un análisis cualitativo en el ámbito universitario. Dirección y Organización.

Bertones D. y Radrigán, M. (2018). Actitudes hacia el emprendimiento: el caso de los estudiantes universitarios chilenos y españoles. Revista de economía pública, social y cooperativa. 1989-6816

Bird, B. (1988). Implementing entrepreneurial ideas: The case for intentions. Academy of Management Review., 13(3).

Bueno, E. (2003). Emprender en la sociedad del conocimiento: el capital del emprendizaje como dinamizador del capital intelectual. En Arnal Losilla (coord.). Creación de empresa: Los mejores textos, Barcelona.

Bustos, V. (2015). Estrategias de aprendizaje, actitudes emprendedoras, auto concepto, esperanza, satisfacción vital e inclinación al autoempleo: Un modelo explicativo en universitarios peruanos.

Cabrera, R. (2005). ¿Por qué es importante la capacitación?. De Gerencia. Recuperado de http://www.degerencia.com/articulo/importante_capacitacion/.

Caicedo, R., Peralta, P., Rodríguez, N., y Quintero, L. (2015). Caracterización del Ecosistema Emprendedor en las Instituciones de Educación Superior en Valledupar, Colombia. Researchgate Recuperado de: https://www.researchgate.net/profile/Aldrin_Carvajal2/publication/308 764256.

Charney, A. y Libecap, G. (2000). The impact of entrepreneurship education: An evaluation of the Berger Entrepreneurship Program at the University of Arizona.

Coduras, A., Urbano, D., Rojas, A., y Martínez, S. (2008). The relationship between university support to entrepreneurship with entrepreneurial activity in Spain: A GEM data based analysis. International Advances in Economic Research, 14. 395-406.

Comisión Europea. Comisión Europea (2003). Libro Verde: El espiritu empresarial en Europa. Bruselas. COM

Durán, S., Parra, M. y Márceles, V. (2015); Potenciación de habilidades para el desarrollo de emprendedores exitosos en el contexto universitario. Serbiluz Sistema de Servicios Bibliotecarios y de Información. Biblioteca Digital Repositorio Académico Opción, 31(77)

\section{Carrera, M.; Partida, A.; Villarreal, L. \& Cantú, A.}


Delicio, F. (2007). La vocación emprendedora de los estudiantes universitarios: El Caso de los estudiantes de la Facultad de Ciencias Económicas y Sociales de la Universidad Nacional de Mar del Plata. Universidad Nacional de Mar del Plata.

Instituto Nacional de Estadística y Grografía (2015). Encuesta Nacional sobre Productividad y Competitividad de las Micro, Pequeñas y Medianas empresas. Disponible en: https://www.inegi.org.mx/programas/enaproce/2015

Espíritu Olmos R. y Sastre Castillo M. (2007). La actitud emprendedora durante la vida académica de los estudiantes universitarios. Cuadernos de Estudios Empresariales, 17. 95-116.

Espíritu Olmos, R. (2011). Actitud emprendedora en los estudiantes universitarios: un análisis de factores explicativos en la Comunidad de Madrid (Tesis Doctoral). Universidad Complutense de Madrid.

Espíritu Olmos R., González Sánchez R. y Alcaraz Vera, E. (2012). Desarrollo de competencias emprendedoras: Un análisis explicativo con estudiantes universitarios. 22. 29-53. Disponible en: http://dx.doi.org/10.5209/rev_CESE.2012.v22.44644.

European Higher Education Area, EHEA (2012) Making the Most of Our Potential: Consolidating the European Higher Education Area. Bucharest Communiqué. Final Version. Disponible

en: http://www.ehea.info/Uploads/(1)/Bucharest\%20Communique\%202012(2).pdf

Fairlie R. y Robb, A. (2007); Families, Human capital, and small business: Evidence from characteristics of business owners survey. Industrial and Labor Relationes Survey, 60.

Fernández, A., Alegre, J., y Chiva, R. (2012). Orientación emprendedora, capacidad de aprendizaje organizativo y desempeño innovador. Journal of technology management \& innovation, 7(2), 157-170.

Fernández, E. \& Junquera, B. (2001). Factores determinantes en la creación de empresas: una revisión de la literatura. Papeles de Economía Española.

Formichella, M. (2004). El concepto de emprendimiento y su relación con la educación, el empleo y el desarrollo local. Buenos Aires, Argentina. Recuperado: http://municipios.unq.edu.ar/modules/mislibros/archivos/MonografiaVersionFinal.pdf

Fontaines, T., Palomo, M. y Velásquez, M. (2015). Resiliencia como Componente de la Actitud Emprendedora de los Jóvenes Universitarios. Revista Educación y Desarrollo Social.

Garavan, T. \& O'Cinneide, B. (1994). Entrepreneurship Education and Training Programmes: a Review and Evaluating. Part I. Journal of European Industrial Training, 18. 3-12.

Gibb, A., Haskins, G. y Robertson, I. (2009). Leading the Entrepreneurial University. Meeting the entrepreneurial development needs of higher education institutions. Recuperado de http://www.ncge.com/publication/leading_the_entrepreneurial_university.pdf.

Glunk, U., y Van Gils, A. (2010). Social Entrepreneurship Education: A Holistic Learning Initiative. International Review of Entrepreneurship, 8(2). 113-131.

González, O. (2004). Educación e iniciativa empresarial: Un análisis centrado en la población juvenil canaria. Colección de soportes audiovisuales e informáticos. Serie Tesis Doctorales del curso 2000-2001. Humanidades y Ciencias Sociales. Servicio de Publicaciones de la Universidad de La Laguna.

\section{Actitudes emprendedoras que inciden en el desarrollo empresarial}


Grichnik, D., Brinckmann, J., Singh, L. y Manigart, S. (2014). Beyond environmental scarcity: Human and social capital as driving forces of bootstrapping activities, Journal of Business Venturing, 29(2). 310-326

Hernández, M. (2020). Fomento de la actitud emprendedora, en el ambiente universitario para la creación de mipymes. Caso de Posgrado de la DACEA-UJAT.

Hernández, R., Fernández, C. y Baptista, p. (2014). Metodología de la Investigación, México.

Ibáñez, M. (2001). Actitudes emprendedoras de los estudiantes universitarios de la CAPV. Tesis doctoral de la Facultad de Ciencias Económicas y Empresariales, Universidad de Deusto, Bilbao, España.

Ibarreche, S. (2019). Management Research. Journal of the Iberoamerican Academy of Management, 17(3), 2019.

World Intellectual Property Organization (2018). Índice Goblal de Innovación. Disponible en: https://www.wipo.int/publications/es/details.jsp?id=4330

Kirzner, I. (1982). The Theory of Entrepreneurship in Economic Growth en Kent, C.A., Sexton, D.L. \& Vesper, K.H. eds. Encyclopedia of Entrepreneurship. Englewood Cliffs, Prentice Hall, Cap.14, pp.272-276.

Krauss, C. (2005). Las actitudes emprendedoras en los estudiantes de la universidad católica de Uruguay. Revista electrónica FCE universidad católica. http://www.ucu.edu.uy/facultades/cienciasempresariales/revistafce/revista6/ artículos/ciela05_enviado_set_(krauss).pdf.

Lanero, A., Vázquez, J. y Muñoz-Adánez, A. (2015). Un modelo social cognitivo de intenciones emprendedoras en estudiantes universitarios. Manuales de psicología, 31(1). 243-259 http://dx.doi.org/10.6018/analesps.31.1.161461

Leite, E.F. O (2012). Fenómeno do Empreendedorismo. São Paulo: Editora Saraiva.

Leite, E, et (2015). El espíritu emprendedor: condicionantes para la innovación. Holos. 278291.

Levine, D. M., Krehbiel, T.C. \& Berenson, M.L. (2006). Estadística para administración. México: PEARSON Educación.

Martín, N., Hernangómez. L. y Rodríguez, A. (2005). Análisis de la Formación y la experiencia Laboral como Determinante del Espiritu Emprendedor de los Estudiantes Universitarios. Revista Asturiana de Economía, 34.

Martínez de Luco, G. y Campos Granados, J. (2014); La intención emprendedora en estudiantes universitarios: El Caso de la Universidad de Deusto. Boletín de Estudios Económicos, 69.

Marulanda., F., Montoya, I., y Vélez, J. (2014). Teorías motivacionales en el estudio del emprendimiento. Pensamiento \& Gestión, 36. 204-236

Monitor Global de Emprendimiento (2018). http://www.deustopulicaciones.es/deusto/pdfs/otraspub/15.pdf

Morales, F., Giménez., J. y Morales, A. (2018). Relaciones entre autoeficacia emprendedora y otras variables psico-educativas en universitarios. European Journal of Investigation in Health, Psychology and Education, 8.

Páez, D., Fernández, I., Basabe, N. y Grad, H. (2002). Valores culturales y motivacionales: Creencias de auto-concepto de Singelis, actitudes de competición de Triandis, control

\section{Carrera, M.; Partida, A.; Villarreal, L. \& Cantú, A.}


emocional e individualismo-colectivismo verticalhorizontal. Revista Española de Motivación y Emoción, 3, 169-175.

Robbins, S. \& Judge, T. (2011). Organizational behavior. (14th) New Jersey: Pearson Prentice Hall.

Robinson, P. (1987). Prediction of entrepreneurship based on an attitude consistency model. Tesis doctoral. Provo, Utah: University of Brigham Young.

Rubio, A. (2012). Juventud, Emprendimiento y Desarrollo: Nuevos nichos de mercado y yacimientos de empleo. Revista de Estudios de Juventud, 99. 35-52.

Quintero, C. (2007). Generación de competencias en jóvenes emprendedores. Primer congreso IPPEU. UNAB. Disponible en: http://www.usmp.edu.pe/facarrhh/ primer_congreso_ippeu_boletin/ppts/Carlos_Quinteros.pdf.

Sánchez, S., Hernández, C. y Jiménez, M. (2016). Análisis de la percepción sobre iniciativa empresarial y el espíritu emprendedor en estudiantes de un tecnológico federal. Acta Universitaria. 26(6). https://doi.org/10.15174/au.2016.1016

Toca, C. (2010). Consideraciones para la formación en emprendimiento: explorando nuevos ámbitos y posibilidades. Estudios Gerenciales, 26(117), 41-60.

Toledano Garrido Nuria (2006). Las perspectivas empresariales de los estudiantes universitarios: un estudio empírico. Revista de Educación, 341. 175-193.

Zapinco, L., Nieto, M. y Muñoz, M. (2008). El coste de oportunidad como determinante del autoempleo en la Unión Europea (UE 25). Pecvnia, 6. 175-193.

\section{Actitudes emprendedoras que inciden en el desarrollo empresarial}

